Vol. 16, Núm. 3

Jul.-Sep. 2020. pp 126-130 doi: $10.35366 / 94525$

https://dx.doi.org/10.35366/94525

\title{
Práctica médica en cirugía de rodilla ante la pandemia COVID-19
}

\author{
Medical practice in knee surgery in the face of the COVID-19 pandemic
}

\begin{abstract}
Roberto F Leal Gómez, ${ }^{*}$ J Alberto Reyes Pedroza, ${ }^{\ddagger}$ Andrés Castillo Álvarez,, Jaime A Armería Vigna," Sandra Archundia Nájera," Miguel Wong Serrano**
\end{abstract}
* Traumatólogo Ortopedista, Subespecialidad en Hombro y Rodilla, Centro Médico Ossis/Ossis Orthosport San Pedro.
‡ Traumatólogo Ortopedista, Subespecialidad en Hombro y Rodilla, Centro de Ortopedia y Medicina del Deporte Puerta de Hierro.
$\S$ MSc. Ortopedia y Traumatología. Fellow, segundo año en Cirugía articular Hombro
y Rodilla, Centro Médico Ossis/Ossis Orthosport San Pedro.
" Traumatólogo Ortopedista, Fellow primer año en cirugía articular de Hombro
y Rodilla, Centro Médico Ossis/Ossis Orthosport San Pedro.
"Traumatólogo Ortopedista, Fellow segundo año en cirugía articular de Hombro y
Rodilla, Centro de Ortopedia y Medicina del Deporte Puerta de Hierro.
** Traumatólogo Ortopedista, Fellow primer año en cirugía articular de Hombro y
Rodilla, Centro de Ortopedia y Medicina del Deporte Puerta de Hierro.

Correspondencia: Roberto F Leal Gómez. Calzada San Pedro No. 804, Col. Fuentes del Valle, 66286, San Pedro Garza García, N.L. Correo electrónico: drrobertoleal@clinicaossis.com

\section{RESUMEN}

Ante la reciente pandemia de coronavirus COVID-19 que enfrentamos es importante tener un panorama general, ya que dicha pandemia ha provocado un gran impacto en nuestra práctica de ortopedia orientada a problemas de rodilla. Las medidas tomadas ante el COVID-19 por distintos hospitales de nuestro país y del extranjero nos han permitido aprender y tomar las nuevas medidas de protección recomendadas por la Secretaría de Salud y la OMS para poder seguir al servicio del paciente. En traumatología ha afectado en distintos niveles como cierre de quirófanos, salas de hospitalización, cancelación de consultas presenciales y suspensión de procedimientos quirúrgicos y de rehabilitación física, teniendo que aumentar la proporción de tratamientos conservadores frente a los quirúrgicos. Acorde con estas

\section{ABSTRACT}

Given the recent coronavirus COVID-19 pandemic that we face, it is important to have an overview since this pandemic has had a great impact on our practice of Orthopedics aimed at knee problems. The measures taken before the COVID-19 by different hospitals in our country and abroad, have allowed us to learn and take the new protection measures recommended by the Secretary of Health and the WHO in order to continue serving the patient. In traumatology, it has affected at different levels, such as the closing of operating theaters, hospitalization rooms, cancellation of face-to-face consultations and suspension of surgical and physical rehabilitation procedures, having to increase the proportion of conservative treatments compared to surgical ones. In accordance 
limitaciones, hemos decidido buscar y compartir los nuevos protocolos implementados para una atención segura al paciente.

Palabras clave: COVID-19, pandemia coronavirus, cirugía de rodilla. with these limitations, we have decided to search for and share the new protocols implemented for safe patient care.

Keywords: COVID-19, coronavirus pandemic, knee surgery.

\section{INTRODUCCIÓN}

Durante la actual contingencia, la patología musculoesquelética no descansa, las fracturas, gonartrosis y gonalgia en general continúan presentándose y los pacientes requieren atención médica especializada. Por este motivo hemos realizado esta revisión de los conceptos actuales en la prevención del contagio con este virus, que afortunadamente se presenta como un cuadro gripal en casi $90 \%$ de los casos, asimismo de los métodos para cuidar a nuestros pacientes al realizar la consulta, exploración física y cirugía. ${ }^{1}$

\section{¿CÓMO PODEMOS PROTEGERNOS Y PROTEGER A NUESTROS PACIENTES?}

Hacer una encuesta al paciente, que puede ser vía telefónica, para determinar si es paciente seguro:

1. Es paciente mayor de 65 años, enfermedad de corazón (hipertensión arterial), diabetes mellitus, historia de cáncer o en tratamiento, enfermedad pulmonar (asma, EPOC), enfermedades que comprometen el sistema de inmunológico (lupus, AR, VIH, trasplantados).

2. Ha tenido contacto con una persona con COVID o personas con síntomas respiratorios.

3. Presenta uno de los siguientes síntomas: fiebre, dolor de cabeza, dolor de garganta o tos, dificultad respiratoria, pérdida de olfato, diarrea.

Si el paciente no cuenta con los factores de riesgo, entonces es un paciente seguro. En caso de que los presente, y su motivo de consulta no sea sumamente urgente, hay que realizar una consulta médica vía telemedicina. ${ }^{2-4}$

\section{Medidas de protección}

- Mantener y respetar una distancia mínima de dos metros.

- Cubrir la boca al estornudars o toser con el ángulo del codo.

- Lavado de manos frecuente, cada hora.

- No tocar la cara con las manos.

- Si es paciente postoperado o subsecuente, hacer telemedicina..$^{2-4}$ 


\section{¿QUÉ PACIENTES PODEMOS VER EN CONSULTA EXTERNA CON PROBLEMAS DE RODILLA?}

Primero hay que determinar el grado de urgencia de la atención, puesto que muchos de los problemas crónicos como la artritis, condromalacia, problemas meniscales degenerativos, problemas de alineación de las extremidades, lesiones ligamentarias, etcétera, se pueden manejar de manera conservadora con analgesia, ejercicios de rehabilitación o cambio de actividad diaria, podemos emplear las alternativas electrónicas disponibles actualmente para monitorear condiciones de la articulación por fotografías o videos y hacer que nuestros rehabilitadores físicos apoyen a los pacientes durante este tiempo desde la oficina. ${ }^{3,4}$

\section{Medidas en la consulta externa}

- Aplicar cuestionario de triage, el cual puede ser vía telefónica por nuestro personal previo a la cita.

- Disminuir el número de consultas por hora para evitar conglomeración en la sala de espera.

- Únicamente se permite un acompañante.

- Distanciamiento social. Mantener dos metros entre personas en sala de espera, la cual está acomodada para respetar dicha distancia.

- No saludar con la mano y mantener distancia.

- Toma de temperatura al ingresar. ${ }^{2-4}$

\section{Medidas de protección obligatorias}

- Pacientes: uso de mascarilla quirúrgica, no de tela, proporcionarla al ingresar al consultorio aunque ya traigan. Lavado de manos (20 segundos) o uso de gel al ingresar y salir de consulta.

- Personal de apoyo en consulta: uso de mascarillas N95 o quirúrgicas, lavado de manos cada hora, gel en seco no más de cuatro veces al día, uso de gafas, lentes o caretas. Asepsia del área donde estuvo el paciente con cloro o soluciones antisépticas (consultorio) y tuvo contacto (sillas, mesas, etcétera) inmediatamente entre paciente y paciente.

- Médicos: uso de mascarilla quirúrgica y guantes, lentes, gafas o caretas, antes y durante valoración, aplicando gel en seco antes y después. Mantener distancia con paciente y realizar exploración física dirigida. ${ }^{5-8}$

\section{¿QUÉ TIPO DE LESIONES DE RODILLA VEMOS EN URGENCIAS?}

- Fracturas, luxaciones, esguinces, bloqueo de rodilla, lesiones ligamentarias, infecciones.

- Todo paciente que se presente a urgencias debe hacer un triage.

- Tomar todas las medidas de protección antes mencionadas.

- Tratar al paciente como si fuera COVID positivo. 
- Definir si requieren de una cirugía inmediata, que de no hacerse comprometería su salud o bien su funcionalidad. ${ }^{9-11}$

\section{¿A QUÉ PACIENTES OPERAMOS?}

\section{Operar pacientes seguros}

A todos los pacientes se les realiza prueba de COVID-19, si son negativos se programan en las siguientes 72 horas después del resultado.

El paciente con problema traumatológico agudo que requiere de tratamiento quirúrgico para mejorar su condición funcional actual o mejorar el dolor que no pudimos resolver de manera conservadora.

$\mathrm{Si}$ el paciente requiere de un tratamiento urgente, se valorará riesgo-beneficio.

Si son problemas ligamentarios, aliviar molestias o realizar artrocentesis y diferir el tiempo prudente. ${ }^{9-11}$

\section{¿SE PUEDE HACER CIRUGÍA ELECTIVA DE RODILLA?}

Se puede hacer cirugía segura a pacientes que no entren en los pacientes de riesgo cuando su problema no pueda diferirse mediante el uso de analgésicos, infiltraciones y/o cambios de actividad, de preferencia en centros de cirugía ambulatoria que no tengan acceso a pacientes con COVID.

Se realiza prueba de COVID a todo paciente que se va a operar para asegurar que no presente una infección asintomática. Si son positivos, se difiere.

Debemos asegurarnos de que las salas de cirugía que se utilicen sólo sean usadas para pacientes seguros, que se limpien de manera exhaustiva, como en casos infecciosos, que se realicen menos cirugías por día y que los pacientes se puedan recuperar en la misma sala de cirugía o separando entre sí las camas de recuperación, con estricto control de personal en quirófano y en general del centro de cirugía seleccionado.

Es preferible diferir a pacientes mayores o con los riesgos anteriormente mencionados cuando haya pasado la contingencia. ${ }^{9-11}$

\section{CONCLUSIONES}

Considerando que esta epidemia no se eliminará rápidamente, debemos aprender a convivir de la mejor manera posible con ella para que nos afecte mínimamente tanto a nuestros pacientes como a nosotros mismos y así poder reiniciar nuestra labor de consulta y cirugía de forma segura.

Debemos tomar especial cuidado en la higiene y en el uso de los métodos de protección y nunca descuidar ni tomar a la ligera el entorno del paciente. Debemos sanitizar el área de consulta y vigilar el tiempo de entrevista con el paciente así como estar totalmente protegidos durante la exploración física, y 
recordar que la mejor manera de no infectarse es no exponernos, tomando lo mejor que hemos aprendido de este problema y aplicándolo de forma rutinaria en nuestras vidas.

\section{BIBLIOGRAFÍA}

1. CDC. Síntomas de la enfermedad del coronavirus. (Internet) [Actualizado 20 de marzo 2020]; [Consultado 28 de abril 2020]. Disponible en: https://espanol.cdc.gov/coronavirus/2019-ncov/symptoms-testing/ symptoms.html.

2. CDC. Evite enfermarse. (Internet) [Actualizado 8 de abril 2020]; [Consultado 28 de abril 2020]. Disponible en: https://espanol.cdc.gov/coronavirus/2019-ncov/prevent-getting-sick/index.html.

3. FEMECOT A.C. Impacto de la pandemia COVID-19: la experiencia en el manejo del paciente. (video file). (25 abril 2020. (02:06:31). [Consultado 28 de abril 2020]. Disponible en: https://www.youtube.com/ watch?v=DoJOAi-xr54.

4. Zbinden-Foncea H, Francaux M, Deldicque L, Hawley J. Does high cardiorespiratory fitness confer some protection against pro-inflamatory responses after infection by SARS-CoV2? Obesity (Silver Spring) 2020; In press. Available in: https://onlinelibrary.wiley.com/doi/epdf/10.1002/oby.22849.

5. Health Protection NSW. COVID-19: Interim guidance for elective surgery and outpatient clinics. (Internet) (Updated March 27, 2020; Consulted April 28, 2020). Available in: https://www.health.nsw.gov.au/ Infectious/covid-19/Pages/elective-outpatient.aspx.

6. American College of Surgeons. COVID-19 Guidelines for triage of Orthopaedic patients. (Internet) (Updated March 24, 2020, Consulted April 28, 2020). Available in: https://www.facs.org/covid-19/ clinical-guidance/elective-case/orthopaedics.

7. AAOS. AAOS Guidelines for elective surgery during the COVID-19 Pandemic. (Internet) (Updated April 2, 2020; Consulted April 28, 2020). Available in: https://www.aaos.org/about/covid-19-information-forour-members/aaos-guidelines-for-elective-surgery/.

8. Becker's ASC Review. ASCA issues guidance for surgery centers amid COVID-19 spread. (Internet) (Updated March 16, 2020; Consulted April 28, 2020). Available in: https://www.beckersasc.com/leadership/asca-issues-guidance-for-surgery-centers-amid-covid-19-spread.html.

9. AAOS. AAOS updates guidelines amid COVID-19 pandemic, including resuming elective surgery. (Internet) (Updated April 22, 2020; Consulted April 28, 2020). Available in: https://www.healio.com/ orthopedics/business-of-orthopedics/news/online/\%7Be8b73a22-d903-4f08-855b-998bc49924f5\%7D/ aaos-updates-guidelines-amid-covid-19-pandemic-including-resuming-elective-surgery.

10. Healthcare Purchasing News. AANA's COVID-19 resources to help keep providers and patients safe during surgery. (Internet) [Updated March 6, 2020; Consulted April 28, 2020]. Available in: https://www. hpnonline.com/surgical-critical-care/article/21128633/aanas-covid19-resources-to-help-keep-providersand-patients-safe-during-surgery.

11. Medscape. Guidelines on Delaying Cancer Surgery During COVID-19. (Internet) (Updated March 26, 2020; Consulted April 28, 2020). Available in: https://www.medscape.com/viewarticle/927568.

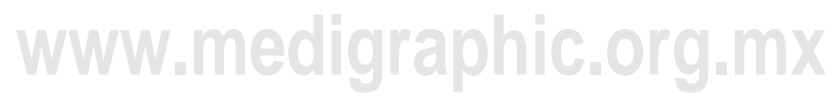

